\title{
Zur Psychodynamik der Kriegshysterie und ihrer Heilung.
}

\author{
Von \\ Karl Landauer (Frankfurt a. M.). \\ (Eingegangen am 3. März 1919. $)^{1}$ )
}

Vorbemerk ung: Diese Arbeit stellt eine direkte Fortsetzung meiner früheren an diesem Orte $^{2}$ ) erschienenen über „Handlungen des Schlafenden" dar: Sie ist aufgebaut auf meinen Beobachtungen als Truppenarzt der Front und der Etappe und bringt so eine - wie ich hoffe - erwünschte Ergänzung zu den Veröffentlichungen aus den Spezialstationen der Heimat. Der Ausgangspunkt der Erörterungen wird deshalb auch ein ganz frischer Fall einer Neurose sein, einer A ktualne urose, die sich in manchen Punkten von den Lazarettprodukten der Heimat unterscheidet, namentlich darin, daß sie die Auslösungs- und Heilungsvor gänge klarer, weil nicht sekundär überarbeitet, zeigt.

Die Krankengeschichte ist unvollständig. Wo und aus welchen Gründen, wird an gegebener Stelle dargelegt werden.

I.

Gefreiter H., 26 Jahre alt, bei der Schwellengewinnungstelle in R. (im südlichen Belgien), im Zivil Fabrikarbeiter.

Vorgeschichte. 1. allgemeine: Eltern und Geschwister gesund. Selbst vor der Militärzeit nie ernstlich krank oder nervös. Kein übermäßiger Alkoholund Tabakgenuß.

2. militärisch: Seit Okt. 1912 aktiv bei Husaren 13. Auğ. 1914 ins Feld. Okt. 1914 leichter Oberschenkelschuß links. 6 Wochen Lazarett. Febr. 1915 erneut ins Feld. Jan. 1916 Diphtherie, 7 Wochen Lazarett. Aug. 1917 als gvE. (wegen Herzbeschwerden) in die Etappe, seitdem verschiedenen Dienststellen zugeteilt. Zur Zeit Fahrer des Offizierswagen der SGSt. R. Seit 25. VIII. $1918 \mathrm{kv}$.

3. Unfall: 30. IX. 1918 etwa 9 Uhr abends (also in der Dämmerung) gingen die Pferde seines leeren Wagens infolge eines entgegenkommenden Autos in einer Ortschaft durch. Er wurde nach etwa $50 \mathrm{~m}$ vor dem Dorfe vom Bock herabgeschleudert und fiel eine Böschung, im ganzen etwa $5 \mathrm{~m}$, herab. Sofortige Bewußtlosigkeit. Wird in diesem Zustand nach 5 Stunden zu mir gebracht.

A uf hah mebefund (1. X. 2 Uhr vormittag): Mittelgroßer kräftiger Mann. Liegt auf der rechten Seite im Bett. Kopf stark nach rechts gedreht. Macht den Eindruck eines Bewußtlosen. Die Lider leisten beim Öffnungsversuch Widerstand. Mehrfaches Ausweichen der Augäpfel nach oben und seitwärts. Vertngerung der Sehlöcher auf Belichtung. Keine äußeren Verletzungen. Große Atmung, etwas röchelnd. Linker Oberarm am Körper anliegend; Ellenbogen und Hand-

1) Durch ein Versehen der militärischen Zınsur blieb die Arbeit mehrere Monate liegen.

2) 41. 1918 . 
gelenk stark gebeugt; Finger in den Grundgelenken leicht gebeugt, in den Zwischenfingergelenken gestreckt, spielen in der Gegend der linken Leistenbeuge (nicht am Genitale). Linkes Bein an allen Gelenken stark gebeugt. Berührungen des linken Armes rufen Stöhnen hervor, ebenso passive Bewegungsversuche im Schultergelenk. Auf deren dreimalige Wiederholung unregelmäßige klonische Zuckungen der ganzen linken Körperseite. Bauchreflexe erhalten, ebenso die anderen normalen Reflexe. Keine Pyramidenzeichen. Psychisch: reagiert nicht auf Anruf, Berührung (außer des linken Armes) und Stiche.

Diagnose: Das Krankheitsbild entspricht weitgehendst dem einer Blutung der motorischen Teile des rechten Gehirns. Die Ähnlichkeit geht bis in Details: Kopfwendung nach rechts bei Lähmung des linken Armes und Zuckungen der linken Körperseite; Spielen der linken Finger; doch fällt hier schon auf, daß das Genitale nicht berührt wird. Dagegen spricht das Fehlen der Pyramidenzeichen, vor allem aber der Widerstand bei der Lidöffnung und das Aufwärts- und Seitwärtsrollen der Augäpfel, also Fluchtbewegungen, die nicht wie beim linken Arm durch Schmerz bedingt sein können. Demnach hysterische Imitation eines Kopftraumas rechts. Dementsprechend wird sofort die

Behandl ung eingeleitet. Ich erkläre laut dem Wärter: Der Mann ist nicht bewußtlos. Er hat keine äußeren oder inneren Verletzungen erlitten. Besprengen Sie ihn mit kaltem Wasser! Er wird dann die Augen aufmachen und seinen Namen nennen. Geschieht. H. schlägt langsam und widerstrebend die Augen auf und sagt auf wiederholte Frage nach seinem Namen sehr laut „Philipp Schmidt“ (,Das ist nicht wahr!"). Leise: „H." (,Ich werde jetzt Ihre Arme und Beine bewegen, damit Sie sich selbst überzeugen, daß nichts gebrochen ist.") Geschieht. Auf weitere Aufforderung hin bewegt er nun alles aktiv im Liegen, steht auf und geht, immer unter energischen Ermahnungen, im Zimmer umher.

Krankheitsverla uf: Schläft die Nacht ruhig. (9 Uhr vormittags): Liegt klar im Bett. Keine äußeren Verletzungen. Kopf, innere Organe und Gliedmaßen außer linkem Arm ohne Befund. Reflexe desgleichen, keine krankhaften. Linker Arm kann angeblich nicht bewegt werden. Zuerst passive Bewegungen, dann nach Kommando immer rascher werdende aktive, zuerst unter erheblichen Stöhnen, Zusammenknicken in den Knien und Äußerungen, daß es nicht gehe, dann immer besser werdend mit mißmutigem Gesichtsausdruck. Nachdem sämtliche Bewegungen (nach 20 Minuten) tadellos ausgeführt werden, erkläre ich, daß er gesund sei. Er könne sich noch etliche Tage ausruhen, dann müsse er wieder zu seiner Truppe. Darauf. Wutausbruch: Er sei kein Schwindler und sei dreimal (!) verwundet gewesen. Auf die erneute Erklärung, niemand halte ihn für einen Schwindler, abermaliges Schimpfen: Der Unfall sei wirklich ernst gewesen.

5 Uhr nachmittags: Klagt über Brustbeschwerden. Ärztliche Versicherung, er sei gesund. Wird zum Arbeitsdienst herangezogen.

2. X. Gibt an, er fühle sich besser, sei nur noch müde. Auf die Versicherung hin, daß er noch e inige Zeit bei der Etap pentruppe sich von seinem Schrecken erholen dürfe, meldet er sich am

3. X. als dienstfähig zur Truppe und bemerkt dazu, er sei froh, so gut weggekommen zu sein.

Auf Befragen trägt er zur Vorgeschichte nach, daß Ph. Schmidt ein Chauffeur und sein Zimmerkamerad in $\mathbf{R}$. sei. Dieser habe Glück: Obwohl er gesünder sei als $H$., sei er wegen Herzleiden bei der letzten Untersuchung auf 6 Monate gvE. geworden, während er selbst kv. geschrieben worden sei und mit seiner Ablö. sung hätte rechnen müssen, wenn ihm der Unfall nicht zugestoßen wäre.

Er sei nicht dreimal verwundet, sondern nur einmal am linken Oberschenkel. Mit der zweiten habe er seine Diphtherie gemeint und mit der dritten seinen jetzigen Unfall. 
Sein jetziger direkter Vorgesetzter, Serg. B., sei dreimal nur ganz leicht vorwundet worden, habe das silberne Verwundetenabzeichen und sei dauernd GvE. Und er mit seiner schweren Diphtherie und seinem lebensgefährlichen Unfall habe gefürchtet, schlechter gestellt zu werden als dieser. Wenn er jedoch seinen Offizierswagen weiter fahren dürfe, sei er schon zufrieden.

Im Lazarett Lille, wo er nach seiner Verwundung kurze Zeit war, sei neben ihm ein Mann mit einem Kopfschuß gelegen, der ,auch" auf der einen Seite ganz gelähmt war, um sich geschlagen habe, und in seiner Gegenwart gestorben sei. Auf Einzelheiten wurde hier wie auch sonst verzichtet, um nicht das Interesse des ja nur sozial Geheilten auf diese heiklen Punkte zu lenken.

2. XI. 1918 stellte sich H. gelegentlich einer Dienstfahrt nach V. bei mir vor; sagte, er habe keine Beschwerden mehr vom Unfall und sprach erneut von dem großen Glück, daß er gehabt habe.

II.

Dies ist alles, was ich von $H$. weiß; wie man sieht, eine recht gewöhnliche Krankheitsgeschichte einer typischen Kriegs- bzw. Unfallhysterie, wie sie jeder Truppenarzt und Neurologe zu Hunderten zu bieten hat. Was sie mir wert zur Veröffentlichung macht, ist gerade ihre Dürftigkeit, die mit scharfen Linien, nicht umrankt von einem schier undurchdringlichen (aber deshalb nicht minder interessanten und erforschungswürdigen) Beiwerk einiges für die Hysterie Charakteristisches hervortreten läßt. Diese Dürftigkeit verdanke ich vor allem dem Mangel an Zeit, welche nur das praktische Ziel der sozialen Heilung erlaubte; zum anderen aber der Tatsache, daß es sich um eine ganz frische Neurose handelte, die bis zur Behandlung erst 5 Stunden dauerte. Sie war deshalb noch nicht in der Lage gewesen, multiple Symptome auszubauen, sozusagen hatte sie nicht Gelegenheit gehabt, an verschiedenen Punkten der Psyche Hilfsanker auszulegen, sondern war nur auf den einen Anker, den sie im Shock geworfen. angewiesen. Es handelte sich demnach noch um eine Aktualneurose.

Nicht ganz mit Recht allerdings kann man hiervon sprechen, da schon früher Herzbeschwerdey bestanden hatten, welche körperlicher Grundlage entbehrten. Wohl mit Sicherheit dürfen diese als neurotische angesehen werden. Auch könnte man über ihre Art leicht sehr wohl begründete Hypothesen aufstellen. Doch unterlasse ich dies und registriere die Herzbeschwerden nur als ein Zeichen, daß $H$. schon früher unter Umständen zu neurotischer Reaktion neigte.

Was zeigt uns nun dieser Fall an?

Das eine ist heute etwas allgemein Anerkanntes: das, was Freud mit „Flucht in die Krankheit“ und „Krankheitsgewinn“, Koh nsta m m mit ,mangelndem Gesundungsgewissen" bezeichnet hat und Kretschmer veranlaßt hat, Hysterie und Simulation gleichzusetzen

$\mathrm{H}$. ist kurz vor dem Unfall $\mathrm{kv}$. geworden und sieht seiner baldigen Ablösung von dem angenehmen Posten und der Etappe entgegen. 
Da ereignet sich der Unfall: Die Pferde gehen in der Dämmerung, geblendet von dem Licht und erschreckt durch den Lärm eines entgegenfahrenden Autos durch. H. wird vom Bock geschleudert und fällt die Straßenböschung, im ganzen etwa $5 \mathrm{~m}$, herab. Ein immerhin sehr beträchtlicher Unfall. Trotzdem kann der Fall als solcher nicht sehr heftig gewesen sein, da jede äußere Verletzung fehlt, ein sehr wichtiger Punkt, dem wir unsere Aufmerksamkeit noch später schenken müssen. Er ist ,,bewußtlos" und bleibt es bis zum Erwecken nach ungefähr 5 Stunden. Aber auch dann ist er nicht der gesunde H., sondern erst der gv. Schmidt, dann der kranke H., der seinen linken Arm nicht bewegt; als er die Lähmung nicht aufrechterhalten kann, schimpft er, beteuert die Schwere des Unfalls, verwahrt sich, Schwindler zu sein; entschuldigt sich also, ohne angeklagt zu sein; lügt im selben Augenblick, er sei dreimal verwundet gewesen; dann hat er Brustbeschwerden. Erst auf die Erklärung des Arztes hin, daß H. sich noch erholen dürfe, daß er seinen Posten in der Etappe behalten könne, meldet er sich als gesund freiwillig zu diesem zurück.

Arzt und H. haben sich damit buchstäblich auf halbem Wege (FrontHeimat) geeinigt, eine für $\mathrm{H}$. und vom militärischen Gesichtspunkt aus gleich befriedigende Lösung.

Aus dieser kurzen Übersicht erhellt auch, warum die meisten Forscher den Ausdruek „,mangelndes Gesundungsgewissen“ oder „Krankheitsgewinn" dem der ,Flucht in die Krankheit" vorziehen. Das, was dem Beobachter des Hysterikers ins Auge fällt, ist, daß der Kranke nicht gesund werden will, also ein sekundäres Moment.

Anders ist es mit der Frage, ob dieser Wille bereits bei der Genese in Betracht kommt, also nach der primären Bedeutung des Willens, aus der Wirklichkeit zu flüchten. Hierüber kann nur der An alytiker (nicht nur der Psychoanalytiker) entscheiden. Wenden wir uns zur Klärung dieser Frage der Entstehung der Neurose zu.

\section{III.}

Man hat häufig eingewandt, daß die Unfälle (mögen es nun „Verschüttungen" oder ähnliche Ereignisse sein) sich so rasch abspielen, daß eine psychische Vorbereitung der auf den Unfall folgenden Erkrankung ausgeschlossen sei.

Nun lag aber an und für sich die Krankheitsbereitschaft bei all unseren Soldaten in der Luft. In den letzten Jahren des Krieges bildete der Wunsch gv. zu werden bzw. zu bleiben, wie man sich allerorten z. B. auf der Straßenbahn oder im Wirtshaus überzeugen konnte, ein Hauptgesprächsthema. Schon um die Jahreswende 1914/15 setzte die offen ausgesprochene Sehnsucht nach dem „Heimatschuß“ oder ähnlichem ein. 
$\mathrm{Daß}$ dem $\mathrm{H}$. solche Wünsche nicht fremd waren, kann man nach den Herzbeschwerden, die er nach der Diphtherie (vielleicht auf Grund einer vorübergehenden Herzmuskelerkrankung) so lange und so gut auszunützen verstand, wohl annehmen. Nun war er kv. geworden. Er rechnete, wie er selbst angab, mit seiner baldigen Ablösung aus der Etappe. Er vergleicht sich mit Schmidt und B. Und sich mit Glücklicheren vergleichen heißt wohl, sein Los verbesserungsfähig finden, und schließt doch auch den Wunsch nach günstiger Wandlung ein.

Da gehen am 30. IX. die Pferde durch.

Nun weiß jeder, der selbst einmal einen Wagen gelenkt oder neben dem Kutscher saß, daß Augenblicke der Spannung dazwischen liegen vom ersten Geräusch eines Autos bis zum Aufschrecken der Pferde, das erst wenige Schritte vor dem Auto erfolgt. Es sind dieselben endlos langen Sekunden wie zwischen dem Abschuß einer Granate und ihrem Einschlag bzw. dem Umschlag des Tones beim „Wegreisen“. Ich habe oft verstandesgemä $B$ einen derartigen $Z$ wischenraum auf 2-3 Sekunden angeben müssen, während es gefühlsmäßig mindestens ebensoviel Minuten waren. Eine Zeit, hinreichend genug, an einen Heimatschuß und an noch viel mehr zu denken. Analog auf einem Pferd oder in einem Wagen zwischen dem Auftauchen und Vorbeisausen des Autos.

Jeder, der mit Pferden umzugehen weiß, kennt auch den Unterschied, den das Verhalten des Reiters bzw. Kutschers in dieser Zeit auf das Pferd hat: ist der Lenker ruhig, so bleibt es meist auch das Pferd. Rettungslos aber teilt sich die Unruhe des Lenkers in erhöhtem Maße dem Gaule mit. Allein der bewußte Gedanke ,hoffentlich passiert nichts", dem bewußt oder unbewußt noch der Wunsch "Schlimmeres" beigefügt ist, genügt: das Pferd wird unruhig, geht durch.

Und zwischen diesem Ereignis und dem des Herabgeschleudertwerdens liegt abermals eine zwar kurze, aber subjektiv endlos lange Zeit. Nun der Sturz. Auch die Fallzeit ist objektiv betrachtet sehr kurz. Der Selbstversuch zeigt jedoch, was währenddessen in uns vorgehen kann bzw. von uns geleistet werden kann, wie das der Sprung von einem Brett von 2-3 m Höhe ins Wasser lehrt.

Diese Utberlegungen geben uns jedoch nur das Recht anzunehmen, daß $H$. sich zum mindesten die letzte Zeit vor dem Unfall mit einem Krankheitswunsche beschäftigt hat. Des ferneren kann man von der Möglichkeit reden, daß ihn in der Spanne bis zum Durchgehen der Pferde (an dem er - vielleicht wenigstens halbbewußt - selbst etwas schuld war) sowie während des Sturzes derselbe Gedanke beseelt haben mag. $\mathrm{Ob}$ dies wirklich der Fall war, könnte nur die Individualanalyse mit Sicherheit beweisen. Diese fehlt aber in unserem Falle.

Immerhin wird die Möglichkeit zur Wahrscheinlichkeit, wenn wir jetzt die Tatsache ins Auge fassen, daß H. 5 m tief herabstürzte und doch 
keine Verletzung erlitt. Das Verständnis dieses auffallenden Gegensatzes zwischen Ursache und Wirkung wird uns durch folgende Beobachtung nahegebracht:

Ioh war am 7. XI. 1918 ans nächster Nähe Augenzeuge folgenden Unfalls: Ein Mann stand, mit dem Abladen eincs Heuwagens beschäftigt, auf ihm in etwa $3 \mathrm{~m}$ Höhe, den Pferd?n abg swandt, als durch die Unvornichtigkeit eines anderen die Pferde plötzlich scheuten und anzogen. Er stürzte halb seitwärts mit ausgebreiteten Armen und Beinen und mußte mit dem linken Arm und Bein unter den Wagen geraten. Da, ganz kurz vor dem Boden und Rad machte er im Sturz eine Wendung, so daß er aufs rechte Knie etwa einen halben Meter seitwärts vom Rade aufschlig und nu" leichte äußere Verletzungen davontrug. Der Mann gab mir sofort an: daß er im Sturz die Gefahr erkannt und dementsprechend gehandelt habe: man wolle doch so kurz vor Schluß (gemeint war der Waffenstillstınd, den wir stündlich erwarteten) nicht noch was abkriegen. Immerhin war der Unfall hier so flötzlich durch eines anderen Schuld hereingebrochen, daß der Zeitpunkt des Falles, welcher mit dem Anziehen der Pferde zusammenfiel, nicht beeinflußbar war. Daher auch nicht die vollständige Rettung. Anders bei H., der nicht sofort im Dorfe, sondern erst an der Böschung, d. h. nach etwa $50 \mathrm{~m}$, vom Wagen stürzte.

IV.

Wir haben uns seither mit der Zeit vor dem Sturze und mit dem Sturze selbst beschäftigt. Nun der Fall, das Aufschlagen.

Hier wendet sich unser Interesse der Shockwirkung zu, welche bei H. weniger eine körperliche, wie die Folgen gezeigt haben, als vielmehr eine seelische war. Betrachten wir zu ihrem Verständnis zunächst einige reinere Fälle, bei welchen die Vorbereitungen fehlten, hierauf solche, bei denen sie kürzer waren:

Im Dezember 1914 wurde auf meinen Verbandsplatz ein Mann gebracht, der nach Angabe der ihn tragenden Kameraden vor etwa einer halben Minute, wenige Schritte entfernt, beim Essenfassen einen Bauchschu $\beta$ durch Infanteriegeschoß erhalten hatte. Der Mann machte zunächst dem Aussehen nach den Eindruck eines Sterbenden: das Gesicht war totenblaß; die Nase trat spitz hervor; kalter Schweiß; röchelnde Atmung; Puls voll, regelmäßig, etwa 70! Einschuß auf dem Mantel etwa in Nabelhöhe. Dem Sitze der Verwundung nach war der Zustand wohl erklärlich, nicht aber der blendend gute Puls. Die Untersuchung ergab, daß das Infanteriegeschoß bei dem Mann, der wegen des kalten Wetters Mantel, Rock, Weste, Sweater, 2 Unterhosen und 2 Hemden anhatte, in den Hemden steckengeblieben war und auf der Haut nur ein kleines rotes Pünktchen, keine Hautverletzung hinterlassen hatte. Als der Mann dies sah, färbte sich sofort wieder sein Gesicht. Er, der soeben wie ein Sterbender dagelegen, ohne ein Glied rühren zu können, sprang ohne Hilfe auf und ging allein zum Essenfassen.

Januar 1915 erhielt mein Bataillonshilfsschreiber, wenige Schritte von dem Unterstand, in dem ich mich befand, einen Brustschuß durch 
Infanteriegeschoß. Er wurde nach etwa 1 Minute als „Sterbender" zu mir gebracht: Einschuß auf dem Rock etwa bei der Herzspitze, Ausschuß auf dem Rücken, so, daß man Herzdurchschuß annehmen mußte. Dementsprechend der Gesamteindruck, Puls jedoch sehr gut. Die Untersuchung ergab einen Tunnelschuß im 4. Zwischenrippenraum. Als ich dies dem Manne mitteilte, und er sich selbst davon überzeugt hatte, konnte er plötzlich allein sitzen und ging dann sofort ohne Hilfe zur etwa $3 \mathrm{~km}$ entfernten Sanitätskompagnie.

Bei einem Vorgehen unseres Nachbarbataillons im Infanteriefeuer sah ich hintereinander 4 Mann schlagartig zusammenstürzen, so daß ich aus der Entfernung von $200-300 \mathrm{~m}$ glaubte, sie seien gefallen. Die Nachschau ergab, daß es sich um lauter leichte Armschüsse handelte.

Uberhaupt ist es eine bekannte Tatsache, daß die Mehrzahl der durch Infanterie Verwundeten hinstürzt, da der Schlag des Treffers ganz plötzlich erfolgt. Selbst wenn nur der Tornister oder ein Kleidungsstück getroffen wird, stürzt in der Regel der Mann, wie ich bei Treffern das eine $\mathrm{Mal}$ an meinem linken Stiefelabsatze, das andere Mal an meiner Mütze selbst erlebte. Immerhin ist dies nicht absolut gesetzmäßig, wie ich bei einem Armschuß des Kollegen J. auf einem nahen Verbandplatze und dem Brustschuß unserer Gefechtsordonnanz beobachten konnte. In diesen beiden Fällen allerdings handelte es sich um verirrte Geschosse, die keine starke Schlagkraft haben, und beide waren während der Zeit der Verwundung durch Gespräch abgelenkt.

Lange nicht so regelmäßig ist das schlagartige Einsetzen des Shockes bei Granat- oder gar Schrapnelltreffer. Hier sieht man viel häufiger die Leute zunächst taumeln und umsinken (nicht umfallen). Hier hört man ja das Herannahen des Geschosses, sieht sich mehr als im Infanteriefeuer der akuten Gefahr gegenüber und ist deshalb vorbereítet.

Während dem plötzlichen Einsetzen des Shockes bei Infanterie, der buchstäblich schlagartig erfolgt, sein ziemlich plötzliches Aufhören entspricht, erfolgt die Lösung des erwarteten Granatshockes in mehr allmählichem Abklingen.

So erhielt einmal Kollege L. durch einen Zünder, den wir heransummen hörten, einen Prellschuß am Unterleib, taumelte und hatte das Gefühl, ohnmächtig zu werden. Noch nach 10 Minuten war ihm "schwummelig", er sah blaß aus und hatle kalten Schweiß. Nach Tagen fühlte er sich noch immer matt und abgeschlagen und war erst sach 5 Tagen wieder ganz hergestellt.

Einen guten Einblick in die subjektiven Vorgänge während eines solchen erwarteten Shocks gibt die Erzählung eines mir befreundeten Offiziers, der vor dem Vorfall schon zweimal verwundet war: Er hatte sich mit seiner Kompagnie aus etwa $500 \mathrm{~m}$ Entfernung auf etwa $150 \mathrm{~m}$ vom Feind herangearbeitet und mußte nun liegenbleiben. Er hatte eine 
kleine Deckung vor sich aufgeworfen. Da sah er gerade vor der Mitte seiner Stirne ein Infanteriegeschoß aufschlagen. Im nächsten Augenblicke würde er tot sein. Starker Schlag über dem Nasenansatz. Er ließ seinen Kopf auf den Boden fallen im Glauben, tot zu sein. Endlose Sekunden. Da habe er sich gefragt, ob denn das wirklich der Tod sei, habe mit der Hand an die Stirne gefaßt und erkannt, daß er nor von einem Lehmstückchen getroffen worden sei, während das Geschoß über ihm weggesprungen sein mußte. Der Herr betonte mir des öfteren, wie lange ihm die Zeit erschienen sei, und wie öde der Gedanke: tot.

V.

Es gibt drei verschiedene Arten von Shock : schlagartig einsetzenden, meist ebenso rasch verschwindenden und den erwarteten, langsam abklingenden. Die dritte Art, den rein körperlichen Shock, schließen wir von diesen Untersuchungen aus. Er besteht zum mindesten in mikroskopischen Zerstörungen der Gehirnmasse. Seine psychische Folge ist (wenn überhaupt eine solche auftritt) die Kommotionsneurose, die sich in einer Abnahme der psychischen Enérgie und im Korsakowschen Syndrom äußert. Im Gegensatz dazu können wir annehmen, daß der schlagartig einsetzende Shock ohne molekulare Gehirnveränderungen verläuft, die nur noch in der Hypothese ganz vereinzelter Forscher (Oppenheim) eine völlig unbewiesene Rolle spielt.

Wir kommen auch ohne deren Annahme sehr gut aus. Stellt doch dieser Shock nur den höchsten Ausdruck dessen dar, was jedem aus eigener Erfahrung als Schreck bekannt ist. Für diesen Affekt sind ja die Empfindungen, daß der Atem stockt, das Herz still steht, die Gliedmaßen starr, kalt und tot sind, charakteristisch. Dazu tritt jenes Gefühl der plötzlichen Leere: die Gedanken stehen still. Mit anderen Worten: es handelt sich um ein plötzliches Totsein.

Schon tief im Tierreich, bei Würmern und vor allem bei Insekten, ist als Reaktion auf Gefahr das Sich-tot-Stellen bekannt. Dieser Reflexvorgang kann in zweierlei Dienste gestellt werden: Einmal der Täuschung des Gegners, zum anderen aber der Selbsttäuschung: alles sei schon vorüber, man ist ja tot. Damit wird die Negation des Ichs zur Negation der Gefahr, ja der gesamten Außenwelt, einschließlich des eigenen Körpers.

Erst die Erkenntnis: ich habe ja keine Schmerzen, es kann also nicht so schlimm sein, bringt z. B. unseren Leutnant dazu, sich wieder der Welt und damit auch seinem eigenen Körper zuzuwenden.

Der Zustand dieses Shocks, oder wie wir ihn auch nennen können: des panischen Schreckens, hat eine Anzahl Berührungspunkte mit dem Schlafe: auch bei diesem besteht eine Abkehr des Ichs von der Außenwelt und vom eigenen körperlichen Ich, das ja zu ihm gehört. Auch hier 
besteht vom Standpunkt des Wachenden aus jene Leere (abgesehen von den Träumen, die aber mit dem Schlaf als solchem nichts zu tun haben).

Allerdings treten beim Shock noch eine Anzahl körperlicher Erzcheinungen dazu wie z. B. die Gänsehaut, nach Darwin eine Reminiszenz an das Sträuben der Felle bei den Tieren.

Der Schreck ist eben nicht Schlaf, wenn auch psychologisch die Analogie mit dem Schlaf sein Verständnis fördert. So ist vor allem der Abschluß von der Außenwelt nicht gleich weitgehend. Zwar bleiben wir auch schlafend mit den geschlossenen Augen, den Hautsinnesorganen und vor allem mit den Ohren in Kontakt mit der Außenwelt. Beim reinen Schreck jedoch werden die Sinnespforten nicht einmal geschlossen, bzw. die Störungen nicht möglichst ausgeschaltet; so bleiben meistens die Augen offen, nur die Gesichtseindrücke sind mehr oder weniger vollständig vom Bewußtsein ferngehalten (daß unbewußt trotzdem viel wahrgenommen wird, ergibt die Befragung in Hypnose). Auch kann, da der reine Shock unvorhergesehen eintritt, nicht eine Lage eingenommen werden, welche Hautsinneseindrücke möglichst fermhält. Endlich ist die Zeit nicht selbst gewählt, d. h.: es wird nicht die Zeit der größten Ruhe, die Nacht, bevorzugt. Der Schlaf bedeutet eben ein Zurückziehen des Ichs auf sich selbst, bildlich in seine Wohnung hinter der Haustüre, während beim Schreck das Ich sich dem störendem Besucher verleugnet: „Ich bin nicht zu Hause."

Der erwartete Shock dagegen ist die Synthese von beiden, von Shock und Schlaf: Schluß der Türe und Verleugnung.

Aus dem Gesagten wird verständlich, warum Leute a uch ohne Disposition zum Abschluß von der Außenwelt, das ist eben zur neurotischen Reaktion, im Kriege erkrankt sind. Ein Mensch, der zum Zurückziehen auf sein Ich neigt, der egozentrisch, autoerotisch oder marzistisch ist, wird zwar leichter auf Gefahr oder auch nur auf Unannehmlichkeiten mit Abschluß des Ichs von der Außenwelt reagieren. Jedoch bestanden allseits sehrstarke Kondition en, wie sie bei H., der typischen Kriegsneurose aufgezeigt wurden, so daß sie auch allein, ohne Disposition, zu einer Neurose führen konnten. Er wartete ja nur darauf, krank zu werden und wurde es ,durch Umsetzung der Gemütsbewegungen in Krankheitserscheinungen, die der Hauptsache nach übertriebenen und verzerrten Ausdrucksformen seelischer Erregungen entsprechen" [Kraepelin $\left.\left.{ }^{\mathbf{1}}\right)\right]$.

Zu den häufigsten Konditionen, die wir auch bei $H$. antreffen, gehört einmal, daß die Neurose in der Dämmerung oder Nacht einsetzt, also in einer Zeit, wo die Welt sich unseren Augen entzieht, wo wir auch

1) Psychiatrie, 8. Aufl., S. 1665.

z. f. d. g. Neur. u. Psych. O. XLviII. 
aus atavistischen und infantilen Erinnerungen mehr die Gefahr fürchten als am Tag, da die Fülle der Eindrücke namentlich aufs Gesicht uns drängt.

Zum anderen ist H. allein. Den Einsamen aber hält nicht Gespräch, nicht Hoffnung auf Hilfe, die von der Außenwelt kommen könnte, an diese mit der Aufmerksamkeit gebannt. Das ist auch der Grund, warum gerade Versprengte, Essenholer, Meldegänger, überhaupt Einzelgeher das Hauptkontingent zu den Neurosen stellen. In der Schützenlinie dagegen entstehen trotz täglicher „Verschüttungen" kaum Neurosen. In Betracht zu ziehen ist, daß dieses Sich-Absondern in der Absicht geschehen kann, die Entstehungsursache der Krankheit zu verschleiern. Dieses schon stark an Simulation grenzende Verfahren ist aber eigentlich nur bei Rezidiven wahrscheinlicher. Anders aber steht es mit dem unbestimmten Gefühl, das viele, die sich aus dem Hexenkessel der Front in die Krankheit flüchten, zuerst ihre Kameraden, an die sie tausend Bande fesseln, verlassen heißt; und die deshalb, um allein zu sein, sich auf irgendeinen Meldegang schicken lassen.

Es wird auch verständlich, warum gerade die Granateinschläge, die sog. ,,Verschüttungen“, in Wirklichkeit das Getroffenwerden mit ein paar Erdstücken, als auslösendes Moment der Hysterien an Häufigkeit so sehr Infanterie- oder Maschinengewehrschädigungen übertreffen. Fast nur beim Granatfeuer bereitet man sich auf akute Gefahr vor und mit der Gefahr auf. die Flucht aus ihr und damit aus der Realität.

Der reine Shock, d. h. der Reflex des Sich-tot-Stellens, ist eine in der menschlichen Gesellschaft recht unpassende und ungeschickte Abwehr der Gefahr. Er ist deshalb nur noch rudimentär, dauert nur noch Bruchteile von Sekunden. Anders, wenn er verbunden ist mit einem freiwilligen Abschluß von der Außenwelt. Hier muß erst die Ursache des Zurückziehens behoben sein. Auch kann die Rückkehr zunächst teilweise erfolgen. So in unserem Fall $\mathbf{H}$.

Diesem Mechanismus der allmählichen Anpassung an die Wirklichkeit, dem Heilungsvorgang haben wir nun nochmals unsere Aufmerksamkeit zu schenken.

\section{VI.}

Die erste Anpassung des $\mathrm{H}$. an die Wirklichkeit aus der vielleicht nur Bruchteile vor Sekunden langen Shockbewußtlosigkeit ist der $\mathrm{Zu}$ stand, in dem er mir gebracht wurde: die hysterische Pseudobewußtlosigkeit.

Sie stellt die Nachahmung eines früher von $H$. beobachteten Kopfschusses dar.

Dies ist etwas Alltägliches; ja die Befragung ähnlicher Kranker in Hypnose oder ihre Erzählung in Analyse ergibt, daß es sich nicht nur um Imitation, sondern um Identifikation handelt. Ich greife aufs 
Geratewohl eine Anzahl ausführlicher Hysteriekrankengeschichten heraus:

Irmal) identifiziert sich mit Frau Professor $N$. in bezug auf das Gefühl, Leichen essen zu müssen, und beißt sich im Dämmerzustand in den Arm, womit sie gleichzeitig sich mit der Toten gleichsetzt (S. 251). Mit dieser auch in anderen somnambulen Anfällen (z. B. S. 217, 242, 284, 289). In anderen Fällen mit der Hexe aus dem Hexenlied von Wildenbruch (S. 272). Im Blutspucken ist sie der Offizier, dessen Blutsturz sie beobachtet hatte (S. 185). Ein anderes Mal die Freundin H. Z., welche ihr vom Zusammensein mit Männern erzählt (Aura der Anfälle, S. 311). Dann ist sie wieder Ophelia, Sappho oder Desdemona (S. 240, 248); dann will sie sich zum Fenster hinausstürzen ,,ich möchte wissen, wie es tut. Ich merke ja gar nicht, daß ich falle. Meine Glieder sind ja heil". Dies, weil sie gesehen, wie ein Mann sich vom Balkon herabstürzen wollte, was sie in Erinnerungstäuschung zu einem Herabstürzen machte (S. 196 und 303).

Marcinowskis Lena ${ }^{2}$ ) identifiziert sich $u$. a. mit Geisteskranken (z. B. S. 585), mit dem Jugendgeliebten, Dr. M. und dem, ,großen Vogel"* (S. 557). Fre uds Dora ${ }^{3}$ ) dagegen mit einer Kusine, die Magenschmerzen bekam (S. 31), mit der Mutter (S. 66), mit einem Vetter, der an Blinddarmentzündung erkrankt ist (S. 89).

Die Identifikation unseres H. mit dem Kopfschuß ist sehr weitgehend, so vollständig, wie es eben ohne Gehirnschädigung möglich ist.

Aber nicht der ganze $\mathrm{H}$. hat sich identifiziert. Mit einem Teil ist er im Kontakt mit der Außenwelt geblieben.

So leisten seine Lider gegen die Öffnungsversuche Widerstand, fliehen die Pupillen, äußert er bei Berührung des linken Armes Schmerz der körperlich begründet sein dürfte. Also zur Erhaltung des Zustandes, der für H. militärisch vorteilhaft ist, ist eine "Wache" abgesondert. Mittels dieser Wache verkehrt der Arzt mit $\mathrm{H}$. Er sagt ihm durch ihre Vermittlung, daß der Zustand wertlos ist (er sei nicht bewußtlos). Durch ihre Vermittlung endlich macht er den Zustand unangenehm durch Bespritzung des H. mit kaltem Wasser.

Daraufhin gibt $\mathrm{H}$. diese Identifizierung zugunsten der neuen mit Ph. Schmidt auf. Hier ist der Abschluß von der Außenwelt nicht mehr so stark: Die Augen sind nun offen, er sieht und hört. Aber er ist als H. immer ,noch nicht zu Hause".

1) L. Binswanger, Versuch einer Hysterieanalyse. Jahrb. f. psychoanalyt. u. psychopath. Forsch. 1, 1909. Deuticke.

2) Heilung eines schweren Falles von Asthma durch Psychoanalyse. Ebenda. $5,1913$.

3) Bruchstück einer Hysterieanalyse. Sammlung kleiner Schriften zur Neurosenlehre 2, 1912. Deuticke. 
Vorher hat das Schlaf-Ich, d. h. derjenige Teil des Ichs, welcher den Abschluß von der Außenwelt wollte, sich zu eben dem Zweck des TraumIchs bedient, indem er ihm die Verfügung über das körperliche Ich überließ. Jetzt beherrscht das Traum-Ich noch den Körper, das Schlaf-Ich ist wieder der Außenwelt zugewandt und diese stellt die Forderung „Sei H.! sei gesund“. Beim ersten festen Zugriff verschwindet nun auch diese Identifikation, der Traumzustand. Noch mehrmals werden ähnliche Versuche vergeblich unternommen. Denn auch die weiteren Symptome stellen sowohl als Krankheit überhaupt, wie als Krankheitszeichen Identifikationen mit anderen Kranken oder mit dem eigenen Ich während früherer Krankheiten dar. Da jedoch diesen Traum-Ichs nie Zeit gelassen wird, sich einzugraben und mit Stacheldraht zu sichern, gelingt es stets leicht, die Symptome zu beheben. Ist ja auch der Krankheitszweck, die Flucht vor der Front, erreicht.

\section{VII.}

Kohnsta m m hat sich damit begnügt, bei der Hysterie eine erhöhte Eignung zur Identifikation anzunehmen, sozusagen als primäres Symptom. Er kommt dazu und muß dazu gelangen, da er nur von einem „mangelndem Gesundungsgewissen" spricht, nicht aber (wie unsere Analyse gezeigt hat) von einer Flucht aus der Realität, einem, wenn auch meist nur partiellen Schlaf.

Wir hatten als die Eigentümlichkeit des Schlafes erkannt, daß das Ich, das schlafen will, das ,Schlaf-Ich“, unter Zurücklassung einer „Wache" sich von der Außenwelt auf das eigene Ich zurückzieht, d. h. auf seine Strebungen aus vergangener Zeit, das ,Traum-Ich“. Damit ist dieses mit all seinen Erinnerungsbildern die Außenwelt des SchlafIchs geworden, die eben als Außenwelt körperlich als Gesichts-, Gehörsusw. Empfindungen projiziert erscheint. Das Schlaf-Ich, in der (abgesehen von der Wache) ausschließlichen Gewalt über das körperliche Ich, steht diesen projizierten Geschehnissen meist als Erlebender gegenüber. Es ist das Gefühl vorhanden : ich träume, oder wenigstens das Ich wird als Zuschauer oder Zuhörer, Handelnder oder Leidender, der während dessen seine Gedanken denkt, dazu empfunden.

Anders, wenn das körperliche Ich von einem Geschehnis betroffen wird, das irgendwelche analogen Erinnerungen an frühere Erlebnisse, das Traum Ich, weckt, und somit das körperliche Ich eine Darstellung des Traum-Ichs wird.

Damit gewinnt dạs Traum-Ich die Herrschaft über das körperliche Ich, ja es hat sie in diesem Augenblick schon. Und wie das körperliche Ich jetzt der Darstellung, der Personifikation des momentanen TraumIchs, das es ursprünglich wachgerufen, dient, wie es mit ihm identifiziert ist, so kann es fernerhin weiteren Identifikationen mit Engrammen 
dienen. $[\mathrm{Da} \beta$ diese energiebeladenen Erinnerungsbilder oft, ja meist nicht von objektiven Erlebnissen, sondern von Phantasien stammen, braucht kaum erwähnt zu werden $\left.{ }^{1}\right)$.]

So in unserem Fall: H. hatte den Wunsch vom Frontdienst verschont zu werden. Es trifft ihn ein Shock, der zum mindesten seit mehreren Sekunden vorausgesehen und erwartet wurde. Der Kopfschuß, den er im Lazarett Lille beobachtet hatte, hatte seine Ruhe. Nun betrifft den Körper des $\mathrm{H}$. ein ähnliches Schicksal, das allerdings nur Bruchteile von Sekunden ihm gleicht.

H. zieht sich von der Wirklichkeit zurück auf sein Traum-Ich, das ist in diesem Augenblick: auf jene Erinnerung an den gestorbenen Kopfschuß, der ihm beneidenswert erscheint und der soeben von seinem Körper dargestellt wird. Er hält erst diese Erinnerung, dann andere, d. h. das Traum-Ich fest und kehrt, da schließlich die Wirklichkeit der Außenwelt annehmbar wird, zu ihr zurück.

Wir haben durch diesen Erklärungsversuch auch die Frage der Umsetzung von Gemütsbewegungen in körperliche Krankheitserscheinungen berührt, eine Eigentümlichkeit, die den meisten Forschern (so Kraepelin und Freud) als ein Charakteristicum der Hysterie erscheint. Wir haben dies wenigstens teilweise auf die Flucht aus der Realität zurückführen können. Ich betone dies ,,teilweise“, da ich mir wohl bewußt bin, daß das Problem viel komplizierter ist. Aber mit den hier zur Verfügung stehenden Mitteln ist es nicht zu lösen. Immerhin ist bereits mit dem Gesagten die Annahme erschüttert, daß die Reaktionsweise primär hysterisch sei.

Als primäres Krankheitssymptom der Hysterie aber haben wir die Flucht aus der Realität erkannt. Jedoch ist dies Symptom nicht charakteristisch für die Hysterie allein; es ist auch der Schlüssel zu anderen Psychoneurosen, ja zu manchen normalen Vorgängen: zum Schlaf und Schreck.

Gerade diese Tatsache zeigt nun eine weitere Unvollständigkeit der Arbeit: Auf die Frage, warum dann H. und die analogen Fälle bei ihrer Flucht aus der Wirklichkeit Hysteriker und nicht z. B. Angstkranke wurden, also auf die Frage der Neurosenwahl, konnte ich hier nicht antworten.

Auf drohende Gefahr nämlich hätte $\mathrm{H}$. ja nicht mit Schreck, sonderı ebensogut auch mit Angst antworten können. Diese aber ist gerade das Gegenteil vom Shock. Einmal körperlich : hier Lähmung des Herzens, der Atmung, der Glieder; dort Herzklopfen, Atembeschleunigung, Zittern. Vor allem psychisch: hier Aufgabe der Außenwelt einschließlich des eigenen körperlichen Ichs; dort erhöhte Zuwendung von Interesse an beide. Also etwa das der Úbere $m$ pfind lich keit Entsprechenda.

$\left.{ }^{1}\right)$ Vgl. Irmas oben angeführte Erinnerungstäuschung: der Sturz vom Balkon. 
262 K. Landauer: Zur Psychodynamik der Kriegshysterie und ihrer Heilung.

Sind es n ur konstitutionelle Momente, die H. den Schreck und damit die Konversionshysterie und nicht die Angst d. n. die Phobie wählen ließen, und welche Konditionen konnten in diesem Fall und können überhaupt eine Rolle spielen?

Die dritte Unvollständigkeit liegt darin, daß die speziellen Symptome unseres Falles längst nicht vollständig geklärt sind: Warum wird z. B. Ph. Schmidt aus der Unzahl der gv. herausgegriffen? Nur weil er seit einiger Zeit Zimmerkamerad des $\mathrm{H}$. ist? Was fesselt ihn sonst an dem Manne? Warum die Brustbeschwerden und nicht z. B. wieder Herzbeschwerden? Zur Lösung all dieser Rätsel hätte ich zur genauen Individualanalyse, zur Psychoanalyse, greifen müssen. Dazu fehlte aber dem Militärarzt das Haupterfordernis: die Zeit. 